\title{
Interaction of Wake and Propulsive Jet Flow of a Generic Space Launcher
}

\author{
Alexander Barklage and Rolf Radespiel
}

\begin{abstract}
This work investigates the interaction of the afterbody flow with the propulsive jet flow on a generic space launcher equipped with two alternative nozzle concepts and different afterbody geometries. The flow phenomena are characterized by experimental measurements and numerical URANS and LES simulations. Investigations concern a configuration with a conventional truncated ideal contour nozzle and a configuration with an unconventional dual-bell nozzle. In order to attenuate the dynamic loads on the nozzle fairing, passive flow control devices at the base of the launcher main body are investigated on the configuration with TIC nozzle. The nozzle Reynolds number and the afterbody geometry are varied for the configuration with dual-bell nozzle. The results for integrated nozzles show a shift of the nozzle pressure ratio for transition from sea-level to altitude mode to significant lower levels. The afterbody geometry is varied including a reattaching and non-reattaching outer flow on the nozzle fairing. Investigations are performed at supersonic outer flow conditions with a Mach number of $M a_{\infty}=3$. It turns out, that a reattachment of the outer flow on the nozzle fairing leads to an unstable nozzle operation.
\end{abstract}

\section{Introduction}

The afterbody flow of a space launcher usually is highly unsteady leading to strong dynamic loads on the nozzle fairing. The wake flow of conventional launcher geometries has already extensively been studied in the literature. Depres et al. [10] performed experiments on a generic launcher geometry with and without a propulsive jet in the transonic flow regime. By evaluating pressure spectra at the base of the launcher they found a distinct peak at a Strouhal number based on the main

\footnotetext{
A. Barklage $(\varangle) \cdot$ R. Radespiel

Institute of Fluid Dynamics TU Braunschweig, Hermann-Blenk-Str. 37,

38108 Braunschweig, Germany

e-mail: a.barklage@tu-bs.de

R. Radespiel

e-mail: r.radespiel@tu-bs.de

(C) The Author(s) 2021 
body diameter $D$ of $S r_{D}=0.2$. The same configuration was investigated by Deck et al. [9] using zonal detached eddy simulations (ZDES) confirming the experimental results by Depres et al.. Stephan et al. [30] performed investigations in the supersonic regime with a propulsive jet. They also observed strong pressure fluctuations at the base at a Strouhal number of $S r_{D}=0.2$. In the hypersonic regime, Saile et al. [22, 23] performed experimental measurements on a generic geometry equipped with a $\mathrm{TIC}^{1}$ nozzle. The same case was investigated by Statnikov et al. [28, 29] using zonal RANS/LES simulations. By performing dynamic mode decomposition they identified dominant modes at $S r_{D}=0.27 ; 0.56$ and 0.85 . Similar modes were also observed by Bolgar et al. [5] on a backward facing step geometry. The aim of reducing structural weight calls for a reduction of these low frequency loads. This can be accomplished by passive flow control devices. Bolgar et al. [6] conducted experimental measurements on a backward facing step equipped with 7 different passive flow control devices. They showed a reduction of the cross-pumping motion of the shear-layer by the lobes. Scharnowski et al. [24] performed measurements on an axisymmetric model equipped with two passive flow control devices in the transonic flow regime. The passive flow control devices lead to reduced pressure and velocity fluctuations downstream of the base. Reedy et al. [20] investigated passive flow control in the form of splitter plates of triangular shape on an axisymmetric bluff body. They carried out experiments at a Mach number of 2,49 and unsteady pressure measurements at the base revealed a reduction of pressure fluctuations of $39 \%$.

However, the above mentioned investigations are restricted to configurations with conventional nozzles and only little knowledge exists concerning unconventional nozzles. One example of unconventional nozzles is the dual-bell nozzle. The dualbell nozzle increases the efficiency of the propulsion system of a space launcher by the use of altitude adaption. The concept was first proposed by Foster and Cowles [8] in 1949. The nozzle features a one-step altitude adaption which is realized by a contour inflection leading to two operation modes, the sea-level mode and the altitude mode. In spite of the advantages from altitude adaption, the dual-bell nozzle encounters performance losses compared to conventional nozzles as have been experimentally measured by Horn and Fisher [15]. Still, the dual-bell offers a payload gain compared to conventional nozzles as has been shown by Stark et al. [27] for an Ariane 5 configuration. Despite these benefits, a dual-bell nozzle was never tested in flight since the transition from sea-level to altitude mode still represents significant uncertainty. During transition high side loads can be generated as observed by Hagemann et al. [14]. Recent studies on a generic space launcher equipped with a dual bell nozzle [3, 4] revealed an unsteady nozzle operation when a supersonic outer flow is present.

This study deals with the wake flow of a generic space launcher and its interaction with the nozzle flow. The focus is on two different configurations with a TIC nozzle and a dual-bell nozzle. The first part concerns the afterbody flow of the TIC configuration and how it can be affected by passive flow control. The second part concentrates on the dual-bell nozzle configuration investigating sensitivity to Reynolds number

${ }^{1}$ TIC: Truncated ideal contour. 
and nozzle fairing length. The length of the fairing is considered as an important parameter for the wake jet flow interaction wherefore an effect on the stability of the nozzle operation is expected.

\section{Experimental and Numerical Setup}

\subsection{Geometry and Test Cases}

The two considered configurations are schematically shown in Fig. 1. Both geometries share the same main body consisting of a cone and a cylindrical part. The cylindrical part has a diameter of $D=108 \mathrm{~mm}$. Downstream of the main body, the geometry features a step decrease in diameter to the diameter of the nozzle fairing, which is $d=61 \mathrm{~mm}$ for the dual-bell nozzle and $d=43 \mathrm{~mm}$ for the TIC nozzle. The model is mounted to the wind tunnel facility by a sword shaped strut support. The base of both geometries can be modified to vary the length of the main body or to apply passive flow control devices. The dual-bell configuration (DB) uses 3 different nozzle fairing length of $l / D=0.85,0.56,0.19$. The investigated flow control devices on the TIC configuration are full-square lobes (FSL) and half-circular lobes (HCL), as seen in Fig. 1. Full-square lobes are chosen since they showed to be most effective in previous studies on a backward facing step [6]. Half-circular lobes are additionally investigated since they produce less wave drag.

The first part of the dual-bell nozzle is designed as a TIC nozzle followed by a constant pressure extension guaranteeing a fast operation mode transition. The transition is calculated to occur at a nozzle pressure ratio of $N P R=p_{0, \text { jet }} / p_{\infty}=$ 12.6 by an transition criterion according to [19], where $p_{0, j e t}$ is the nozzle total pressure. A detailed description of the dual-bell nozzle is found in [1]. The nozzle of the TIC configuration features the same throat diameter as the dual-bell nozzle of $25.31 \mathrm{~mm}$. The Mach number at the nozzle exit is $M a_{\text {exit }, n z}=2.5$. For a detailed description of the TIC nozzle the reader is referred to [30].

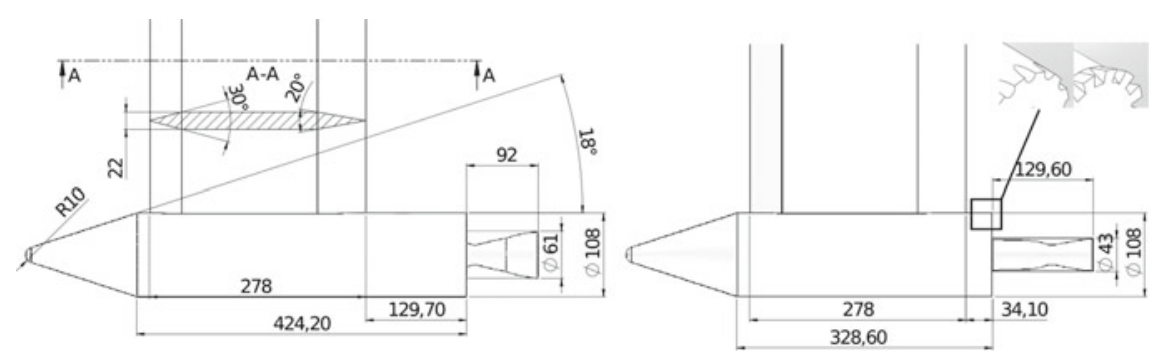

Fig. 1 Geometry of the investigated launcher models. Dual-bell nozzle configuration (left) and TIC configuration with and without lobes (right). Dimensions are in Millimeters 
Table 1 Flow conditions of ambient and jet flow

\begin{tabular}{l|l|l|l|l|l|l|l}
\hline & $M a_{\infty}$ & \multicolumn{2}{|l|}{ Ambient flow } & \multicolumn{2}{l}{ Jet flow } \\
\cline { 3 - 8 } & & $R e_{\infty, D}$ & $T_{0, \infty}(\mathrm{K})$ & $p_{\infty}(\mathrm{Pa})$ & $N P R$ & $R e^{*}$ & $T_{0, \text { jet }}(\mathrm{K})$ \\
\hline $\begin{array}{l}\text { TIC, flow } \\
\text { control }\end{array}$ & 3 & $1.3 \cdot 10^{6}$ & 285 & 4,100 & 97.6 & $1.5 \cdot 10^{6}$ & 285 \\
\hline $\begin{array}{l}\mathrm{DB}, R e \\
\text { variation }\end{array}$ & 0.1 & - & 285 & $\begin{array}{l}1,300- \\
31,000\end{array}$ & $10-20$ & $\begin{array}{l}0.2- \\
12.3 \cdot 10^{5}\end{array}$ & 285 \\
\hline $\begin{array}{l}\mathrm{DB}, l / D \\
\text { variation }\end{array}$ & 3 & $2.5 \cdot 10^{6}$ & 285 & 7,900 & $5-10$ & $\begin{array}{l}1.5- \\
3.0 \cdot 10^{5}\end{array}$ & 285 \\
\hline
\end{tabular}

The flow conditions differ for the three different investigations. The corresponding flow conditions are listed in Table 1 . The working gas for ambient and jet flow is dry air at a temperature of $T=285 \mathrm{~K}$. The outer flow is supersonic with a Mach number of $M a_{\infty}=3$ for the cases where the base geometry is varied, whereas no outer flow is considered for the variation of nozzle Reynolds number. Numerical simulations use a Mach number of $M a_{\infty}=0.1$ for the case without an outer flow since a compressible flow solver is used. The nozzle total conditions are constant in the experimental measurements while the numerical simulations also allow time dependent nozzle total conditions.

The flow control cases consider an under-expanded jet flow with $N P R=97.6$. The lobe configuration is referred to as 'valley' if there is a lobe valley located $\varphi=180^{\circ}$ and as 'peak' if there is a lobe peak at $\varphi=180^{\circ}$.

For the variation of nozzle Reynolds number $R e^{*}=\left(\rho^{*} u^{*} D^{*}\right) / \mu^{* 2}$ the nozzle pressure range $N P R$ is kept constant while increasing the ambient pressure. Numerical simulations were performed for two different ambient pressures of $p_{\infty}=4,100 \mathrm{~Pa}$ and $p_{\infty}=16,400 \mathrm{~Pa}$ which will be referred to as 'low Reynolds number case' and 'high Reynolds number case', respectively. Experiments are conducted for a wider range of Reynolds numbers.

The variation of $l / D$ features an increased outer flow Reynolds number compared to the flow control case since this guarantees turbulent nozzle flow conditions, as will be shown later.

\subsection{Experimental Setup}

\subsubsection{Wind Tunnel and Jet Simulation Facility}

Experimental measurements are conducted in the Ludwieg tube wind tunnel of TU Braunschweig (HLB) in its supersonic configuration. The wind tunnel consists of

\footnotetext{
${ }^{2}$ Star values correspond to nozzle throat conditions, where: $\rho^{*}:$ Density, $u^{*}$ : Velocity in x-direction, $D^{*}$ : Throat diameter.
} 
a high pressure part which is the $17 \mathrm{~m}$ long storage tube and a low pressure part which contains a settling chamber, the wind tunnel nozzle and a vacuum tank. A fast acting valve separates these two parts. The high pressure part can be pressurized up to 30 bar and the low pressure part is evacuated to a few millibars prior to a wind tunnel run. The wind tunnel run starts with the opening of the fast acting valve. The air is then accelerated by the wind tunnel nozzle so that the Mach number in the test section is $M a_{\infty}=2.9$. The flow conditions in the test section are constant for approximately $50 \mathrm{~ms}$ and the maximum achievable unit Reynolds number is $R e=35.3 \cdot 10^{6} 1 / \mathrm{m}$. The original design of the wind tunnel is described in [11] and the supersonic configuration is discussed in [32, 33].

The jet simulation facility $\left(\mathrm{TSA}^{3}\right)$ provides pressurized gas for the jet flow. The TSA also uses the ludwieg tube working principle. The $32 \mathrm{~m}$ long storage tube is connected from outside of the wind tunnel to the launcher model. The storage tube can be pressurized up to 160 bar and heated up to $900 \mathrm{~K}$. The model itself contains the fast acting valve as well as a settling chamber and the dual-bell nozzle. The TSA is operated simultaneously with the wind tunnel and constant nozzle flow conditions are achieved for approximately $100 \mathrm{~ms}$. The reader is referred to [30] for a detailed discussion on the TSA.

\subsubsection{Instrumentation}

The afterbody of both configurations is equipped with 3 time resolving pressure sensors while the dual-bell configuration additionally features 7 pressure sensors on the nozzle wall. The used sensors are Kulite XCQ-062 with a natural frequency of $150 \mathrm{kHz}$. Figure 2 shows an overview on the instrumentation. The base of both configurations is equipped with one sensor at $(r / D, \varphi)=\left(0.42,180^{\circ}\right)$ with the strut support being at $\varphi=0^{\circ}$. Two pressure sensors are located on the nozzle fairing at $(x / D, \varphi)=\left(0.04,180^{\circ}\right)$ and $(x / D, \varphi)=\left(0.51,180^{\circ}\right)$ for the dual-bell configuration $^{4}$ and at $(x / D, \varphi)=\left(0.31,180^{\circ}\right)$ and $(x / D, \varphi)=\left(0.77,180^{\circ}\right)$ for the TIC configuration. The nozzle wall of the dual-bell configuration is instrumented with 7 flush mounted pressure sensors at four axial positions of $x / D=0.44,0.5,0.56,0.73$ and at three different circumferential positions of $\varphi=0^{\circ}, 90^{\circ}, 180^{\circ}$. A Spectrum M2i.4652 recorder samples the pressure data at a rate of $3 \mathrm{MHz}$. Boundary layer tripping was applied slightly downstream of the nozzle throat at $x / D=0.21$.

Schlieren imaging is used for a qualitative characterization of the flow topology. The schlieren configuration is a conventional coincident configuration using a gas discharge lamp as light source. A Phantom v711 camera records the schlieren images at a recording frequency of $13,000 \mathrm{~Hz}$ and a resolution of $800 \times 600$. The exposure time is $2 \mu \mathrm{s}$.

\footnotetext{
${ }^{3}$ German abbreviation for 'Treibstrahl Simulations Anlage'.

${ }^{4}$ All axial positions of the dual-bell configuration are measured from the base in the $1 / \mathrm{D}=0.85$ configuration.
} 

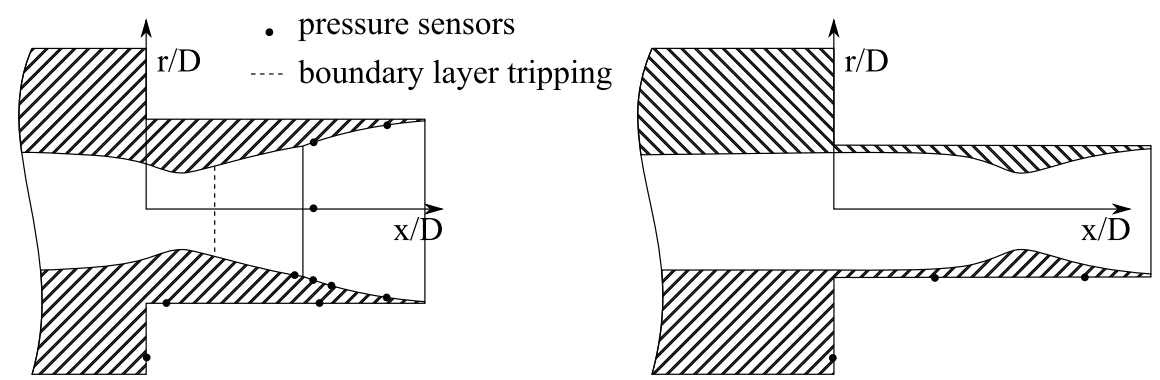

Fig. 2 Instrumentation of the afterbody of the launcher model. Dual-bell configuration with a nozzle length of $x / D=0.85$ (left) and TIC configuration without lobes (right)

\subsection{Numerical Setup}

\subsubsection{URANS Setup}

Numerical simulations use the DLR TAU code [25] for performing unsteady Reynolds averaged Navier-Stokes computations. In this study, the governing equations represent a two-dimensional axisymmetric formulation of the Navier-Stokes equations. The solver uses a finite-volume scheme in a conservative formulation. The one-equation turbulence model of Spalart and Almaras [26] closes the RANS equations. Laminar solutions are achieved by setting the Reynolds stresses to zero. Viscous terms are approximated by a central scheme of second order accuracy. Inviscid terms are discretized by the AUSMDV upwind scheme of Wada and Liu [31]. Temporal discretization uses an implicit euler dual-time stepping scheme of second order accuracy with a time step size of $1 \cdot 10^{-6} \mathrm{~s}$.

This study uses two computational grids, both being two-dimensional and axisymmetric with a circumferential extent of $\Delta \varphi=1^{\circ}$. The grid for cases without an outer flow (grid 1) contains the nozzle wall and the fairing but does not cover the main body and counts 190, 000 cells. The grid for cases with an outer flow (grid 2) also covers the main body, however neglecting the strut support and counts 220,000 cells. Walls are modeled as isothermal with a wall temperature of $293 \mathrm{~K}$ applying a no-slip condition. At the nozzle inlet time varying reservoir conditions are applied with a linearly varying total pressure with a slope of $\Delta N P R / \Delta t_{\text {ref }}=680.351 / \mathrm{s}$ with $t_{\text {ref }}=t u_{\infty} / D$. A gradient of zero for the flow variables realizes a symmetrical axis at $r / D=0$. The boundaries in circumferential direction assume axisymetric flow. The remaining boundary conditions of grid 1 represent farfield conditions. Grid 2 uses a supersonic inflow conditions at the upstream boundary and a supersonic outflow condition at the downstream boundary. 


\subsubsection{Zonal RANS/LES Setup}

Zonal RANS/LES computations were carried out using the flow solver developed at the Institute of Aerodynamics of RWTH Aachen University [18]. The solver uses a finite volume scheme in conservative formulation to solve the compressible NavierStokes equations. The solver is capable of performing RANS and LES simulations as well as coupled zonal RANS/LES simulations using a Reformulated Synthetic Turbulence Generation (RSTG) method [21]. Sub-grid scales in the LES formulation are modeled by the monotone integrated LES (MILES) method [7]. A one-equation turbulence model of Fares and Schröder [12] closes the RANS equations. Spatial discretization employs second order accurate central differences for viscous terms and a second order accurate mixed centered upwind AUSM scheme [16] for inviscid terms. Temporal integration uses a second order accurate explicit 5-stage RungeKutta method. The solver is detailed in [17].

This work features two computational grids for investigating the Reynolds number influence corresponding to the low Reynolds number and high Reynolds number conditions, respectively. Both conditions share the same RANS grid with a total size of about one million cells. The LES grid counts 77 million cells for the low Reynolds number condition and 277 million cells for the high Reynolds number condition. The RANS solution is coupled to the LES solution by performing an independent solution and then creating a database for interpolation onto the LES boundary. The coupling position is slightly upstream of the contour inflection to guarantee an established boundary layer at the inflection point. The RANS domain employs reservoir conditions with a linear varying total pressure at the nozzle inlet. The slope of the time varying pressure is chosen accordingly to the URANS configuration from Sect. 2.3.1. Walls are modeled as adiabatic using a no-slip condition. All remaining boundaries are farfield conditions employing a characteristic approach. A detailed description of the grids can be found in [1].

\section{Results}

\subsection{Passive Flow Control on TIC Configuration}

The effectiveness of the two lobe configurations is evaluated based on the induced mechanical loads on the nozzle fairing. The mean pressure distribution characterizes static loads whereas pressure fluctuations characterize dynamical loads. The mean pressure distribution is shown in Fig. 3 on the left hand side. All cases show a similar trend with a downstream increase in pressure due to the reattaching shear layer that forms at the shoulder of the main body. At the base, there is a recirculation region which is differently pronounced for the clean case and the lobe configurations. The pressure at the base is lower for the lobe cases compared to the clean case indicating a stronger expansion at the shoulder of the main body. A stronger expansion 

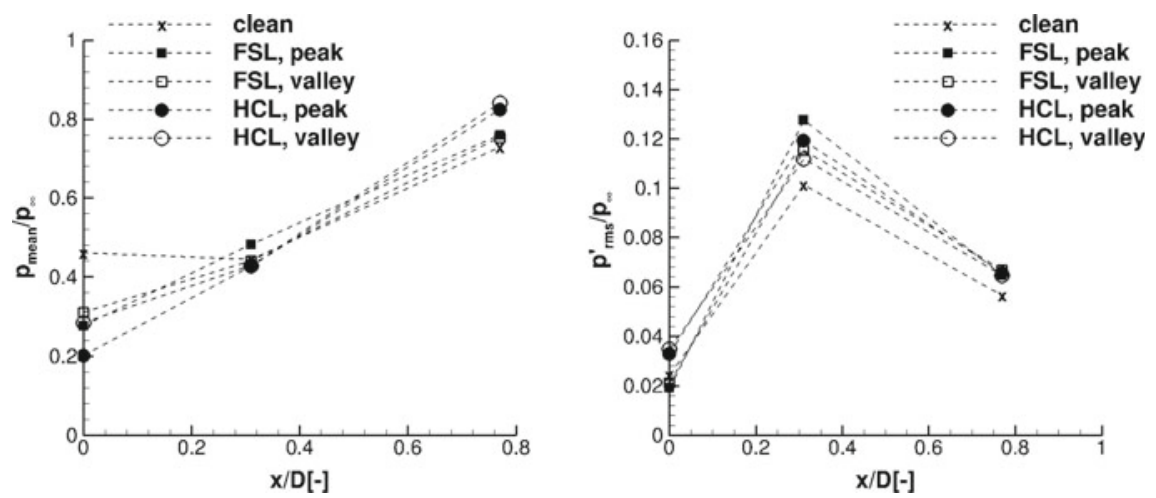

Fig. 3 Pressure distribution (left) and pressure fluctuation (right) on the nozzle fairing for clean case and lobe configurations

corresponds to a stronger deflection of the shear layer at the shoulder wherefore the lobes reduce the recirculation region.

However, the influence of the lobes diminishes downstream of the base where the pressure is slightly increased compared to the clean case regardless of the lobe configuration. This corresponds to a shift of the reattachment shock slightly further upstream. The influence on dynamic loads can be seen in Fig. 3 on the right hand side. Again, all configurations show a similar trend with lowest fluctuations at the base, which increase further downstream, followed by a decrease. The fluctuations at $x / D=0.31$ are highest since this point lies in the reattachment region where the shear layer impinges on the nozzle fairing. Further downstream, the flow is attached leading to reduced pressure fluctuations. The lobes are shifting the pressure fluctuations to higher values compared to the clean case thus increasing the dynamical loads on the fairing. The higher fluctuations might be explained by the streamwise vortices induced by the lobes. The lobe configuration shows a minor influence and the full-square lobes lead to lower fluctuations at the base and higher fluctuations at $x / D=0.31$ compared to the half-circular lobes. This is in contrast to findings at subsonic Mach numbers by Bolgar et al. [6]. Lobes seem not to be useful for suppressing dynamical loads at supersonic flow conditions on axisymmetric geometries.

\subsection{Analysis of Dual-Bell Transition-Effect of Reynolds Number}

The aim of this chapter is to determine a Reynolds number range where sub-scale dual-bell investigations are comparable to a full-scale application. For full-scale applications the boundary layer of the nozzle flow is always turbulent, wherefore laminar flow should be avoided in sub-scale investigations. Reynolds number ranges 

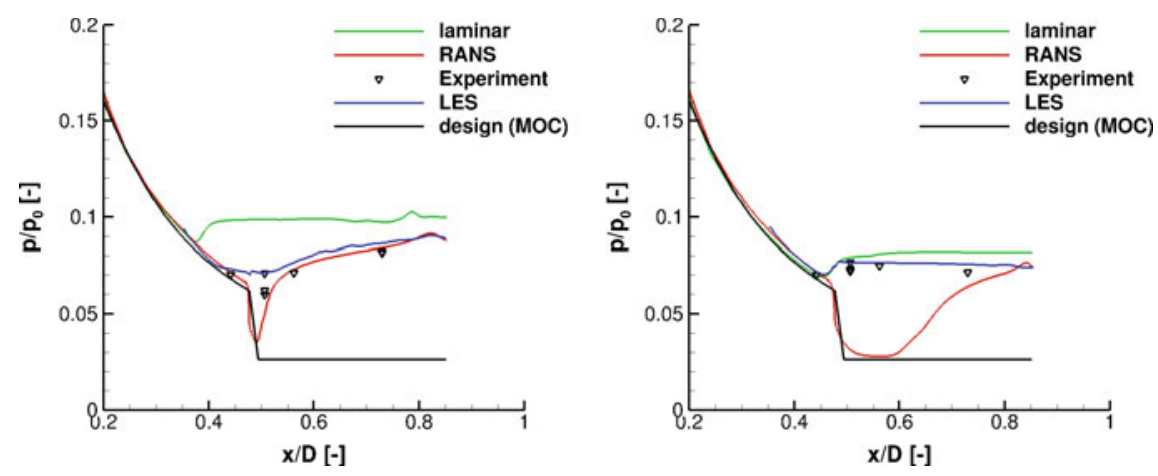

Fig. 4 Pressure distribution on the nozzle wall for $N P R=10$ at high Reynolds number condition (left) and for $N P R=12$ at low Reynolds number condition (right)

for laminar and turbulent nozzle flow are therefore determined. The 'low Reynolds number' case represents laminar flow conditions while the 'high Reynolds number' case represents turbulent flow conditions. Figure 4 shows a comparison of numerical and experimental results in terms of pressure distribution in the nozzle extension. Additionally, the design values are shown which are determined by the method of characteristics (MOC) corresponding to the values in altitude mode since attached flow is assumed. For the high Reynolds number case there is a good agreement between measurements and numerical simulations. The nozzle operates in sea-level mode at $N P R=10$ what is characterized by an increase of pressure compared to the design value downstream of the inflection point at $x / D=0.47$. The laminar solution shows a stronger separation and deviates from the measurements and the RANS and LES solutions indicating this case to be turbulent. The low Reynolds number case reveals a different behavior and the laminar solution agrees well with the LES solution and the measurements. The RANS solution deviates from the measurements and the nozzle flow separates in the nozzle extension at approximately $x / D=0.63$. Since the laminar solution, the LES simulation and the measurements show consistent results, the low Reynolds number case corresponds to laminar conditions.

In order to characterize the nozzle transition process, unsteady numerical simulations were carried out covering a transition followed by a retransition. The resulting separation position is summarized in Fig. 5 on the left hand side. The condition for determining the separation position is that the friction coefficient equals zero $\left(c_{f}\left(x_{\text {sep }}\right)=0\right)$. It is noted, that LES results are averaged in circumferential direction. The LES results show a strong Reynolds number influence as the $N P R$-value for transition is strongly increased for the low Reynolds number case compared to the high Reynolds number case. The laminar solution predicts transition in a similar N P R-range for the low Reynolds number case, revealing this case to be laminar. The LES solution for the high Reynolds number case is considered as turbulent since it better compares with the URANS solution. The URANS solution however shows no significant Reynolds number influence. Figure 5 right shows a comparison of 

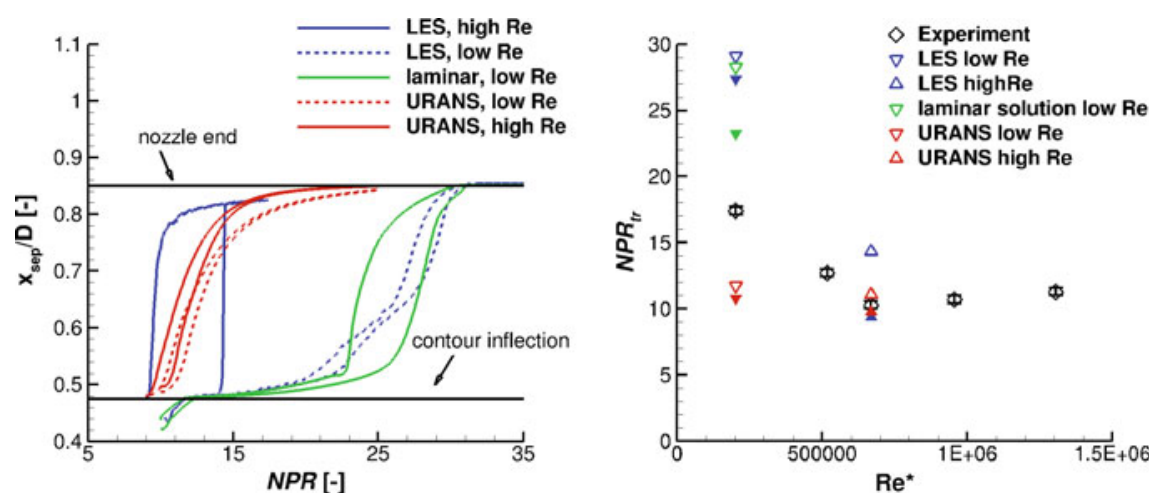

Fig. 5 Position of flow separation for the numerical simulations (left) and $N P R$ value for transition as a function of nozzle Reynolds number $R e^{*}$ (right), open symbols stand for transition and filled symbols for retransition

the $N P R$-value for transition and retransition compared to experimental data. This value is determined for the simulations at the point where the value $d x_{\text {sep }} / d N P R$ reaches its maximum and for the measurements by successively increasing the nozzle total pressure until a switch to altitude mode is observed. The value of $N P R_{t r}$ for the high Reynolds number case matches well between the simulations and measurements whereas for the low Reynolds number case there is a discrepancy. This discrepancy might be related to a transitional boundary layer state in the measurements. The measurements reveal that for $R e>6,68 \cdot 10^{5}$ there is no significant change in $N P R_{t r}$ wherefore this Reynolds number range is regarded as turbulent. As shown in Ref. [2] the Reynolds number range for turbulent flow can be extended to $R e>1,40 \cdot 10^{5}$ by using boundary layer tripping, wherefore tripping is used in the following.

\subsection{Analysis of Dual-Bell Transition-Influence of Afterbody Geometry}

This section deals with the dynamic interaction of the supersonic outer flow with the dual-bell nozzle flow for different afterbody geometries. There already exist studies on the influence of the nozzle fairing length on dynamic loads for generic launcher geometries equipped with TIC nozzles [10, 13]. Van Gent et al. [13] found that for a nozzle length where no reattachment on the nozzle fairing occurred, there is a pronounced interaction of the plume and the outer flow. For a dual-bell configuration, it was previously shown in [3], that an unstable nozzle operation occurs for a configuration with $l / D=1.37$. In this case, the nozzle alternately switched between altitude mode and sea-level mode at a distinct frequency. The frequency of this flipflop mode is evaluated from the pressure signals of a sensor located in the nozzle extension at $x / D=0.56$ for the different afterbody geometries and different values 

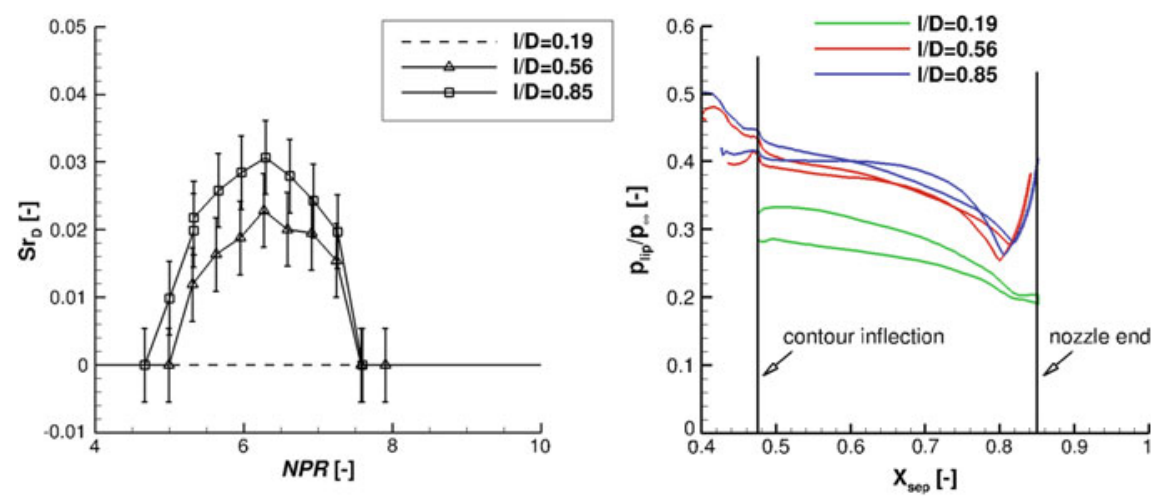

Fig. 6 Flip-flop frequency as a function of $N P R$ (left) and variation of the lip pressure $p_{\text {lip }}$ during transition and retransition from URANS simulations (right)

of $N P R$. These results are summarized in Fig. 6 on the left hand side. The Strouhal number $S r_{D}$ is computed based on the main body diameter $D$ and the freestream velocity $u_{\infty}=607 \mathrm{~m} / \mathrm{s}$. Error bars of frequency are related to the minimum resolvable frequency due to a time window size of $\Delta t=40 \mathrm{~ms}$ of the pressure signals. No flip-flop mode occurred for the configuration with $l / D=0.19$, wherefore this curve always equals zero. The other configurations feature a flip-flop mode for a certain $N P R$ range which is approximately $\triangle N P R=4.7-7.6$ for $l / D=0.85$ and $\triangle N P R=5-7.6$ for $l / D=0.56$. The maximum flip-flop frequency is reached at $N P R \approx 6.3$ for both configurations though the frequency is lower for $l / D=0.56$ compared to $l / D=0.85$. These results show that the nozzle operation is more stable with decreased fairing length and for a certain fairing length of $0.19 \leq l / D<0.56$ the flip-flop mode completely diminishes.

The flip-flop mode appears to be triggered by a variation in back pressure since the nozzle transition is determined by the pressure ratio between nozzle total pressure and back pressure. The back pressure equals the ambient pressure for the case without an outer flow whereas the back pressure with a supersonic outer flow is altered by the expansion of the outer flow at the nozzle lip. Hence, the pressure at the nozzle lip $p_{l i p}$ is relevant for the transition and retransition process. The value of $p_{l i p}$ from the URANS simulations during transition and retransition is shown in Fig. 6 as a function of the separation position. The configurations $l / D=0.85$ an $l / D=0.56$ exhibit a minimum which is not present for $l / D=0.19$. This behavior is important to characterize the stability of the nozzle operation since a negative value of $d p_{l i p} / d X_{\text {sep }}$ favors transition and a positive value favors retransition. For example, a negative value means that as the transition moves to the nozzle end, the back pressure decreases thus supporting the transition. For $l / D=0.19$ there is always a negative value of $d p_{\text {lip }} / d X_{\text {sep }}$ considering this case as stable. In contrast, the cases with $l / D=0.85$ and $l / D=0.56$ feature a negative value for the interval $0.48 \leq x / D<0.81$ and 

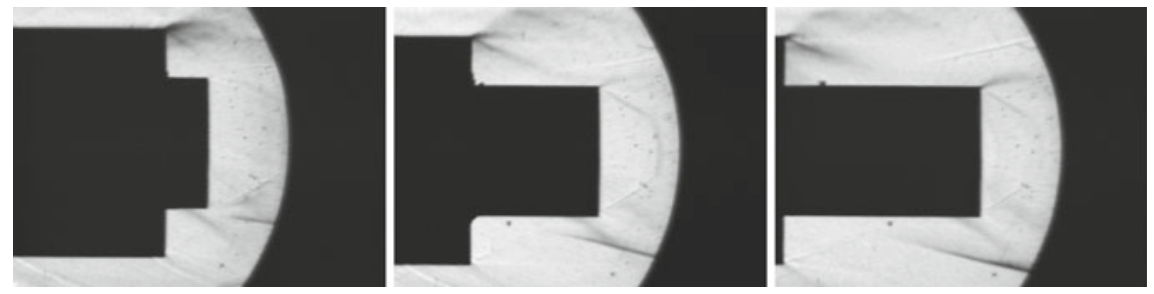

Fig. 7 Schlieren images at altitude mode for $l / D=0.19 l / D=0.56 l / D=0.85$ (from left to right)

a positive value for the interval $0.81<x / D \leq 0.85$, considering these cases to be unstable.

The schlieren images in Fig. 7 give an overview on the flow topology of the three configurations at altitude mode operation. For all three cases the outer flow separates at the shoulder of the main body and the shear layer bends towards the nozzle fairing. The shear layer reattaches on the fairing for the cases with $l / D=0.85$ and $l / D=0.56$ characterized by a reattachment shock. Further downstream, the flow expands at the nozzle lip and a shear layer develops between nozzle flow and outer flow. For the case with $l / D=0.19$ there is no reattachment of the outer flow on the nozzle fairing, instead the outer flow reattaches on the nozzle flow featuring a reattachment shock and a shear layer. The occurrence of the flip-flop mode in the supersonic regime is therefore related to the reattachment of the outer flow on the nozzle fairing. A reattaching flow leads to an unstable operation while a nonreattaching flow leads to a stable operation.

\section{Summary}

This paper summarizes three studies on a generic space launcher equipped with a TIC nozzle and a dual-bell nozzle, respectively. These studies include experimental as well as numerical investigations.

The effectiveness of passive flow control devices was investigated on the TIC configuration. It turns out, that both passive flow devices under consideration lead to a reduced pressure at the base corresponding to a shorter recirculation region compared to the clean configuration. However, the lobes lead to moderately increased dynamical loads on the nozzle fairing as they increase pressure fluctuations.

The nozzle Reynolds number was varied for the dual-bell configuration in order to determine corresponding Reynolds number ranges for turbulent and laminar flow conditions. The nozzle flow reveals to be turbulent for a Reynolds number range of $R e^{*}>6.68 \cdot 10^{5}$. By using transition tape, the Reynolds number range of turbulent flow can be extended to $R e^{*}>1.40 \cdot 10^{5}$ so that investigations with outer flow and turbulent nozzle conditions can be performed in the present wind tunnel setup. 
Studies of a dual-bell nozzle interacting with an outer flow showed a instable nozzle operation featuring the so called flip-flop mode which is characterized by an alternating switch between sea-level and altitude mode. The flip-flop mode revealed to be sensitive to the length of the nozzle fairing. It diminishes if the outer flow does not reattach on the nozzle fairing.

Further studies will concern a second dual-bell nozzle design with an increased hysteresis gap compared to the nozzle used in this studies. Hot wire measurements will be also performed in the wake to correlate fluctuations in the outer flow with the unsteady nozzle behavior.

Acknowledgements Financial support has been provided by the German Research Foundation (Deutsche Forschungsgemeinschaft - DFG) in the framework of the Sonderforschungsbereich Transregio 40. Computational resources have been provided by the High Performance Computing Center Stuttgart (HLRS) and by the North-German Supercomputing Alliance (HLRN). The authors gratefully acknowledge Chloé Génin for designing and providing the contour of the used Dual-Bell nozzle.

\section{References}

1. Barklage, A., Loosen, S., Schröder, W., Radespiel, R.: Reynolds number influence on the hysteresis behavior of a dual-bell nozzle. In: Proceedings of the 8th European Conference for Aerospace Sciences (EUCASS), pp. 1-11. EUCASS2019-519 (2019)

2. Barklage, A., Radespiel, R.: Influence of the boundary layer state on the transition of a dual-bell nozzle. Deutsche Gesellschaft für Luft- und Raumfahrt - Lilienthal-Oberth e.V. (2019)

3. Barklage, A., Radespiel, R., Génin, C.: Afterbody jet interaction of a dual-bell nozzle in supersonic flow. In: AIAA Propulsion and Energy Forum. American Institute of Aeronautics and Astronautics (2018). AIAA Paper 2018-4468

4. Bolgar, I., Scharnowski, S., Kähler, C.: Experimental analysis of the interaction between a dualbell nozzle with an external flow field aft of a backward-facing step. In: Tagungsband des 21 . DGLR-Fachsymposium der STAB, Notes on Numerical Fluid Mechanics and Multidisciplinary Design. Deutsche Gesellschaft für Luft- und Raumfahrt, Cham, Switzerland, 2019 (2019)

5. Bolgar, I., Scharnowski, S., Kähler, C.J.: The effect of the mach number on a turbulent backward-facing step flow. Flow, Turbul. Combust., pp. 1-28 (2018)

6. Bolgar, I., Scharnowski, S., Kähler, C.J.: Passive flow control for reduced load dynamics aft of a backward-facing step. AIAA J. 57(1), 120-131 (2019)

7. Boris, J., Grinstein, F., Oran, E., Kolbe, R.: New insights into large eddy simulation. Fluid Dyn. Res. 10(4-6), 199-228 (1992)

8. Cowles, F.B., Foster, C.R.: Experimental study of gas-flow separation in overexpanded exhaust nozzles for rocket motors. JPL Progress report, pp. 4-103 (1949)

9. Deck, S., Thorigny, P.: Unsteadiness of an axisymmetric separating-reattaching flow: Numerical investigation. Phys. Fluids 19, 1-20 (2007)

10. Deprés, D., Reijasse, P.: Analysis of unsteadiness in afterbody transonic flows. AIAA J. 42(12), 2541-2550 (2004)

11. Estorf, M., Wolf, T., Radespiel, R.: Experimental and numerical investigations on the operation of the hypersonic ludwieg tube braunschweig. In: Fifth European Symposium on Aerothermodynamics for Space Vehicles, vol. 563, p. 579 (2005)

12. Fares, E., Schröder, W.: A general one-equation turbulence model for free shear and wallbounded flows. Flow, Turbul. Combust. 73(3-4), 187-215 (2005) 
13. van Gent, P.L., Payanda, Q., Brust, S.G., van Oudheusden, B.W., Schrijer, F.F.J.: Eects of exhaust plume and nozzle length on compressible base flows. AIAA Journal 57(3), 1184-1199 (2019)

14. Hagemann, G., Terhardt, M., Haeseler, D.: Experimental and analytical design verification of the dual-bell concept. J. Propuls. Power 18(1), 116-122 (2002)

15. Horn, M., Fisher, S.: Dual-bell altitude compensating nozzles. Technical report, NASA (1994)

16. Liou, M.S., Steffen, C.J.: A new flux splitting scheme. J. Comput. Phys. 107(1), 23-39 (1993)

17. Meinke, M.: Numerische lösung der navier-stokes-gleichungen für instationäre strömungen mit hilfe der mehrgittermethode. Ph.D. thesis, RWTH Aachen University (1993)

18. Meinke, M., Schröder, W., Krause, E., Rister, T.: A comparison of second-and sixth-order methods for large-eddy simulations. Comput. Fluids 31(4-7), 695-718 (2002)

19. Nürnberger-Génin, C., Stark, R.: Experimental study on flow transition in dual bell nozzles. J. Propuls. Power 26(3), 497-502 (2010)

20. Reedy, T.M., Elliott, G.S., Dutton, J.C., Lee, Y.: Passive control of high-speed separated flows using splitter plates. AIAA J. 50(7), 1586-1595 (2012)

21. Roidl, B., Meinke, M., Schröder, W.: A reformulated synthetic turbulence generation method for a zonal rans-les method and its application to zero-pressure gradient boundary layers. Int. J. Heat Fluid Flow 44, 28-40 (2013)

22. Saile, D., Guelhan, A.: Plume-induced effects on the near-wake region of a generic space launcher geometry. In: Proceedings of the 32nd AIAA Applied Aerodynamics Conference, AIAA, vol. 3137, p. 2014 (2014)

23. Saile, D., Gülhan, A., Henckels, A., Glatzer, C., Statnikov, V., Meinke, M.: Investigations on the turbulent wake of a generic space launcher geometry in the hypersonic flow regime. In: Progress in Flight Physics, vol. 5, pp. 209-234. EDP Sciences (2013)

24. Scharnowski, S., Bosyk, M., Schrijer, F.F.J., van Oudheusden, B.W.: Passive flow control for the load reduction of transonic launcher afterbodies. AIAA J. 57(5), 1818-1825 (2019)

25. Schwamborn, D., Gerhold, T., Heinrich, R.: The DLR TAU-Code: recent applications in research and industry. In: Proceedings of the European Conference on Computational Fluid Dynamics (ECCOMAS) (2006)

26. Spalart, P.R., Allmaras, S.R.: A one-equation turbulence model for aerodynamic flows. In: 30th Aerospace Sciences Meeting and Exhibit (1992). AIAA Paper 92-0439

27. Stark, R., Génin, C., Schneider, D., Fromm, C.: Ariane 5 performance optimization using dual-bell nozzle extension. J. Spacecr. Rocket. 53(4), 743-750 (2016)

28. Statnikov, V., Sayadi, T., Meinke, M., Schmid, P., Schröder, W.: Analysis of pressure perturbation sources on a generic space launcher after-body in supersonic flow using zonal turbulence modeling and dynamic mode decomposition. Phys. Fluids 27(1), 016,103 (2015)

29. Statnikov, V., Stephan, S., Pausch, K., Meinke, M., Radespiel, R., Schröder, W.: Experimental and numerical investigations of the turbulent wake flow of a generic space launcher at $m_{\infty}=3$ and $m_{\infty}=6$. CEAS Space J. 8(2), 101-116 (2016)

30. Stephan, S., Wu, J., Radespiel, R.: Propulsive jet influence on generic launcher base flow. CEAS Space J. 7, 453-473 (2015)

31. Wada, Y., Liou, M.S.: A flux splitting scheme with high-resolution and robustness for discontinuities. In: 32nd Aerospace Sciences Meeting and Exhibit (1994). AIAA Paper 94-0083

32. Wu, J., Radespiel, R.: Tandem nozzle supersonic wind tunnel design. Int. J. Eng. Syst. Model. Simul. 47 5(1-3), 8-18 (2013)

33. Wu, J., Radespiel, R.: Experimental investigation of a newly designed supersonic wind tunnel. In: Progress in Flight Physics, vol. 7, pp. 123-144. EDP Sciences (2015) 
Open Access This chapter is licensed under the terms of the Creative Commons Attribution 4.0 International License (http://creativecommons.org/licenses/by/4.0/), which permits use, sharing, adaptation, distribution and reproduction in any medium or format, as long as you give appropriate credit to the original author(s) and the source, provide a link to the Creative Commons license and indicate if changes were made.

The images or other third party material in this chapter are included in the chapter's Creative Commons license, unless indicated otherwise in a credit line to the material. If material is not included in the chapter's Creative Commons license and your intended use is not permitted by statutory regulation or exceeds the permitted use, you will need to obtain permission directly from the copyright holder. 